BGP-15 treated group when compared to the control group. No significant differences were revealed between anti-collagen antibody levels or in the distribution of T-cells, B-cells, dendritic cells and monocyte/macrophages harvested from draining lymph nodes, suggesting an effect predominantly involving the innate immune system.

Conclusions Our results demonstrate that the novel chaperoneinducer BGP-15 has a profound prophylactic and therapeutic effect on autoimmune arthritis, likely due to an effect on the effector phase.

\section{A9.10 NEUTRALISATION OF ACPA - A WAY TO GO?}

doi:10.1136/annrheumdis-2013-203223.10

${ }^{1} \mathrm{C}$ Cerqueira, 'E Ossipova, 'M Hansson, 'L Mathsson, 'L Klareskog, ${ }^{2} \mathrm{~J}$ Rönnelid, 'P-J Jakobsson. 'Department of Medicine, Unit of Rheumatology, Karolinska University Hospital, Stockholm, Sweden; '2Department of Immunology, Genetics and Pathology, Uppsala University, Uppsala, Sweden

Background and Objectives In a previous study, we have identified endogeneously citrullinated sites in fibrinogen from RA synovial tissue (Hermansson, et al, 2010 in Proteomics-Clin Appl). Within the alpha chain, Arg573 and Arg591 were found citrullinated with an occupancy rate in the range of $1-2 \%$ and in the $\beta$-chain, Arg72 and Arg74 were also found citrullinated. We now demonstrate that these citrullinated residues are autoantigenic as well as demonstrate that peptides containing these epitopes can be used as probes for development of ACPA neutralising compounds.

Materials and Methods The autoantigenic potential was investigated using the Phadia's ImmunoCAP ISAC $®$ system. Citrullinated and unmodified fibrinogen peptides were immobilised onto a glass slide in an arrayed fashion and serum from 404 CCP positive and 532 CCP negative RA patients and 461 healthy controls from the EIRA cohort were tested. We also assayed the identified citrulline fibrinogen peptides for their ability to prevent purified ACPA (Ossipova, et al, 2012 submitted) to bind to CCP (CCPlus ${ }^{\circledR}$ ELISA, Euro-Diagnostica $A B)$. Peptides were individually or in combinations incubated with different ACPA pools and the blocking efficiency was expressed as percent of inhibition and IC50. Corresponding arginine peptides were used as controls.

Results We found that $31 \%$ ( $87 \%$ are CCP positive) of patients were positive to Cit573 peptide. For the Cit591 peptide, the corresponding numbers were $10 \%$ (65\%), for the Cit74 peptide $28 \%$ $(68 \%)$ and for the Cit72 peptide $20 \%$ (68\%). Interestingly, citrullinated 573 and Cit591 peptides revealed a maximum of $77 \%$ and $48 \%$ ACPA inhibition, respectively. When equally mixed, these peptides displayed an additive higher degree of ACPA neutralisation (84\%). In contrast, Cit74 and Cit72 peptides reached a more modest maximum inhibition of $26 \%$ and $30 \%$, respectively. This experiment was repeated using a different set of ACPA pool and then the efficiencies were lower for Cit573 (47\%) but similar for Cit591 (51\%). Logically, the efficiency of specific citrullinated compounds will depend on the individual ACPA specificities.

Conclusions Here we demonstrate extensive autoantibody reactivity against in vivo citrullinated fibrinogen epitopes found in RA synovial membranes. These peptides can now be used as additional biomarkers for studies of ACPA sub-specificity profiles as recently reported (Brink, et al, $2012 A \& R$, in press). We also demonstrate that these citrullinated peptides can be used as neutralising agents blocking a significant portion of ACPA binding to CCP. These results open novel possibilities for the design of personalised ACPA blockers preventing for instance the osteoclastogenesis and bone loss induced by ACPA (Harre, et al, 2012 JCI).

\section{A9.11 NF-kB INDUCING KINASE (NIK) IS A KEY REGULATOR OF INFLAMMITION-INDUCED ANGIOGENESIS}

doi:10.1136/annrheumdis-2013-203223.11

'AR Noort, 'KPM van Zoest, ${ }^{2 P}$ Koolwijk, 'PP Tak, 'SW Tas. 'Division of Clinical Immunology and Rheumatology, Academic Medical Center, University of Amsterdam, The Netherlands; 'Department of Physiology, Institute for Cardiovascular Research (ICaR-VU), VU University Medical Center, Amsterdam, The Netherlands

Background In rheumatoid arthritis (RA) synovial tissue (ST) angiogenesis can be observed already in the earliest phase of disease. The chemokine CXCL12, which is induced via the non-canonical nuclear factor-kappaB (NF-kB) pathway, plays an important role in angiogenesis, lymphocyte transendothelial migration, and the homing of endothelial progenitor cells. Therefore, the non-canonical pathway, with its key mediator NF-kB inducing kinase (NIK), may play an important role in pathological angiogenesis and the perpetuation of synovial inflammation in RA.

Objective To study the role of non-canonical NF-kB signalling in pathological angiogenesis in RA.

Methods Expression of NIK and CXCL12 in RA ST was evaluated using immunofluorescence microscopy (IF). Angiogenesis was studied in endothelial cells (EC) in vitro using the tube formation assay and ex vivo by comparing WT and $\mathrm{NIK}^{-/}$mice in the aortic ring assay. Physiological (developmental) angiogenesis was evaluated by isolectin B4 staining of the retina followed by confocal microscopy. The contribution of NIK to synovial angiogenesis was studied in vivo in antigen-induced arthritis (AIA).

Results NIK, p52 and CXCL12 were highly expressed in EC in RA ST, mainly in small (newly formed) blood vessels. Stimuli that induce noncanonical NF-kB signalling (lymphotoxin (LT), LIGHT, and CD40L) significantly enhanced in vitro tube formation 2.5-fold $(p<0.05)$, which could be completely blocked by siRNA targeting NIK or IKK $\alpha$. Aortic rings from WT and NIK/- mice showed normal TNF- and VEGFinduced microvessel outgrowth. In contrast, whereas non-canonical NF-kB stimuli induced microvessel outgrowth in WT mice (unstim $29.94 \pm 6.08$ versus LT $159.1 \pm 50.24$ versus LIGHT $110.3 \pm 17.68$ $\left.\left(\mathrm{mm}^{2}\right) \mathrm{p}<0.05\right)$, no microvessel outgrowth was observed in aortic rings from $\mathrm{NIK}^{-/-}$mice (unstim $28.74 \pm 15.89$ versus $\mathrm{LT} 45.9 \pm 16.71$ versus LIGHT $\left.43.41 \pm 15.73\left(\mathrm{~mm}^{2}\right)\right)$. In line with this, $\mathrm{NIK}^{-1}$ mice exhibited normal developmental angiogenesis in the retina, but a $50 \%$ reduction in pathological angiogenesis in synovial inflammation (blood vessels in synovial tissue WT $20 \pm 5.07$ versus $\mathrm{NIK}^{-1} 10.2 \pm 3.02$ ).

Conclusions NIK is preferentially expressed in EC in RA ST and non-canonical NF-kB signalling in EC results in enhanced angiogenesis in vitro. NIK $\%$ mice exhibited normal developmental and VEGFinduced angiogenesis, but reduced pathological angiogenesis in AIA. These findings point towards an important role of the non-canonical NF-kB pathway in pathological angiogenesis associated with chronic (synovial) inflammation. This could be exploited for the development of future new therapies for $R A$.

\section{A9.12 NON-CANONICAL NF-KAPPAB SIGNALING INDUCES A PROANGIOGENIC RESPONSE IN RHEUMATOID ARTHRITIS SYNOVIAL FIBROBLASTS AND ENDOTHELIAL CELLS}

doi:10.1136/annrheumdis-2013-203223.12

${ }^{1,2} \mathrm{CX}$ Maracle, ${ }^{1,2} \mathrm{AR}$ Noort, ${ }^{1,2} \mathrm{KPM}$ van Zoest, ${ }^{1,2} \mathrm{SW}$ Tas. ${ }^{1}$ Divison of Clinical Immunology and Rheumatology; ${ }^{2}$ Department of Experimental Immunology, Academic Medical Center/University of Amsterdam, Amsterdam, Netherlands

Background Synovial fibroblasts (SF) play a pivotal role in the pathophysiology of rheumatoid arthritis (RA). RASF not only promote inflammation and cartilage destruction of the joint, but also stimulate angiogenesis. Angiogenesis allows for increased leukocyte 